\title{
RATE OF EXHAUSTION OF A CLOSED TANK BY A RECIPROCATING AIR PUMP.
}

By Edgar Buckingham.

ABSTRACT.

An equation is deduced for finding the number of strokes of a reciprocating air pump required to exhaust a tank of large volume down to any given fraction of atmospheric pressure, assuming that the piston and valves are tight. The resulting formula shows how clearance and valve loading affect the speed of exhaustion and the final minimum attainable pressure.

\section{CONTENTS.}

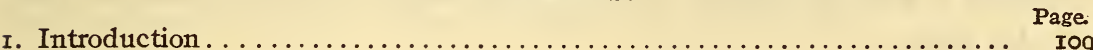

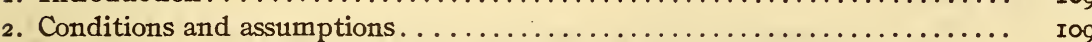

3. Notation . . . . . . . . . . . . . . . . . . . . . . . . . . . .

4. The reduction of pressure per cycle of the pump $\ldots \ldots \ldots \ldots \ldots \ldots \ldots \ldots$ III

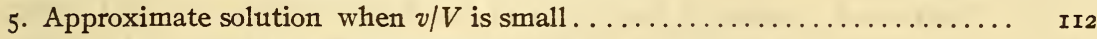

6. Approximate forms of the equation . . . . . . . . . . II

7. Numerical examples............................. II5

\section{INTRODUCTION.}

A letter recently referred to the writer asked for a formula for computing the rate at which a reciprocating air pump would exhaust an air-tight tank of known volume. After an unsuccessful search through several books which might have been expected to contain such a formula, an equation was developed for the purpose; and since it may be of interest to some readers, the deduction is given below.

Any attempt to allow for leakage past valves and piston would require the use of special hypotheses, which might not correspond at all closely to actual working conditions, or, if such hypotheses were dispensed with, would lead to a result which was too general and indefinite to be of any value. Hence it is postulated, from the start, that valves and piston shall be in perfect condition and the whole system free from leaks. The remaining conditions for the validity of the result are stated in the next section.

\section{CONDITIONS AND ASSUMPTIONS.}

(a) The tank to be exhausted is large in comparison with the piston displacement of the pump, so that each pump cycle reduces the pressure in the tank by only a very small fraction of itself 
(b) The exhaustion is slow enough that the temperature in the tank remains sensibly constant, the pressure in the tank being then proportional to the amount of air remaining.

(c) The process starts with atmospheric pressure in the tank, and the pump discharges to the atmosphere.

(d) There is a constant load on the suction valve and no throttling except at the valve. This means that at the end of any suction stroke the pressure in the pump cylinder is less than that in the tank by a small constant amount which is determined by the load on the valve but is independent of the degree of exhaustion attained.

(e) The temperature of the air in the pump at the end of any suction stroke is constant, regardless of the degree of exhaustion. Usually it will, of course, be somewhat higher than the temperature in the tank.

( $f$ ) The compression line on the pump diagram follows the usual equation $p v^{\mathrm{n}}=$ constant, where $\mathrm{I}<n<\mathrm{I} .4$.

(g) There is a constant load on the discharge valve, so that when the valve closes at the end of any compression stroke, the pressure in the clearance space is a small constant amount above the outside atmospheric pressure to which the pump is discharging.

\section{NOTATION.}

Let $V=$ the volume to be exhausted;

$v=$ the piston displacement of the pump;

$\epsilon=$ the fractional clearance, so that when the piston is full out the cylinder volume is $(I+\epsilon) v$;

$\theta_{0}=$ the absolute temperature of the air in the tank;

$\theta=$ the absolute temperature of the air in the pump cylinder at the end of a suction stroke;

$P=$ the outside atmospheric pressure to which the air is discharged;

$\alpha P=$ the load on the discharge valve, so that the valve lifts when the pressure in the pump has risen to $(I+\alpha) P$; $\beta P=$ the load on the suction valve;

$p=$ the pressure in the tank at the end of any suction stroke, the pressure in the pump being then $p-\beta P$;

$n=$ the exponent of the compression line-it is between I and I.4;

$x=p / P=$ the degree of exhaustion reached;

$N=$ the number of suction strokes since the start at $x=\mathrm{I}$;

$l=$ the lowest or limiting value of $x$, for $N=\infty$. 


\section{THE REDUCTION OF PRESSURE PER CYCLE OF THE PUMP.}

We start at the end of a suction stroke, when the pressure is $p$ in the tank and $(p-\beta P)$ in the pump cylinder. If $M=$ the mass of air in the tank and $m=$ the mass in the cylinder, we have

$$
M=\frac{p V}{R \theta_{0}}
$$

and

$$
m=\frac{(p-\beta P)(\mathrm{I}+\epsilon) v}{R \theta}
$$

where $R$ is the gas constant for unit mass.

Let $w=$ the volume under the piston at the instant when the discharge valve lifts. Then we have, by hypothesis,

whence

$$
(P+\alpha P) w^{n}=(p-\beta P)[(\mathrm{I}+\epsilon) v]^{n}
$$

$$
w=(\mathrm{I}+\epsilon) v\left(\frac{p-\beta P}{P+\alpha P}\right)^{\frac{1}{n}} .
$$

When the discharge valve closes at the end of the compression stroke, the volume is $\epsilon v$. The pressure, in the clearance space is the same as when the valve opened, and we shall assume that the temperature is sensibly the same; hence the mass of air remaining in the clearance space is $m \epsilon v / w$ or, by equations (2) and (3),

$$
m \frac{\epsilon v}{v}=\frac{\epsilon v}{R \theta}(P+\alpha P) \frac{x}{n}(p-\beta P) \frac{n-1}{n} .
$$

The piston now moves out, the suction valve opens, and more air flows from the tank to the cylinder. If $\Delta p=$ the resulting decrease of the pressure in the tank, the pressure in the cylinder at the end of this suction stroke is $(p-\beta P-\Delta p)$, and the mass contained in the cylinder is (see equation 2)

$$
m^{\prime}=\frac{(p-\beta P-\Delta p)(\mathrm{I}+\epsilon) v}{R \theta}
$$

of which the mass $m \epsilon v / w$ given by equation (4) was already present before the suction valve opened. The mass taken from the tank during this suction stroke is therefore

$$
\Delta M=m^{\prime}-m \frac{\epsilon v}{w}
$$


or, by equations (4) and (5),

$$
\Delta M=\frac{(\mathrm{I}+\epsilon) v}{R \theta}\left[p-\beta P-\Delta p-\frac{\epsilon}{\mathrm{I}+\epsilon}(P+\alpha P) \frac{x}{n}(p-\beta P) \frac{n-\mathrm{I}}{n}\right]
$$

But since, by hypothesis, the temperature in the tank is constant, the pressure changes in the same ratio as the mass of air and we have $\Delta p / p=\Delta M / M$, or, by equations (I) and (6),

$$
\frac{\Delta p}{p}=\frac{(\mathrm{I}+\epsilon) \theta_{0} v}{p \theta V}\left[p-\beta P-\Delta p-\frac{\epsilon}{\mathrm{I}+\epsilon}(P+\alpha P) \frac{\mathrm{I}}{n}(p-\beta P)^{\frac{n-\mathrm{I}}{n}}\right] \text { (7) }
$$

Let

$$
\frac{(\mathrm{I}+\epsilon) \theta_{0}}{\theta V} \equiv g ; \frac{\mathrm{I}+\epsilon}{\epsilon}(P+\alpha P) \frac{\mathrm{I}}{n} \equiv h
$$

and equation (7) may be reduced to the form

$$
\frac{\Delta p}{p-\beta P}=\frac{g v}{\mathrm{I}+g v}\left[\mathrm{I}-\frac{h}{(p-\beta P) \frac{\mathrm{x}}{n}}\right]
$$

where $g$ and $h$ are constants defined by equation (8).

\section{APPROXIMATE SOLUTION WHEN $\nabla / V$ IS SMALL.}

Equation (9) gives the reduction $\Delta p$ of the pressure in the tank during one complete cycle of the pump, starting with the pressure p. By successive computations, the total reduction caused by any number of cycles could be found, but such a process would be very laborious. It must therefore be replaced, if possible, by a single computation covering any desired number of strokes taken together.

The actual process of exhaustion proceeds by finite steps, but if we postulate that $v / V$ shall be small, these steps will be small, and after a certain number of suction strokes $N$, during which the total piston displacement is $N v=\varphi$, the pressure in the tank will be very nearly the same as if this total displacement had been divided into a much larger number of strokes of a correspondingly smaller pump.

We may therefore get an approximately correct result by supposing the pump volume $v$ to be infinitesimal; so we replace $v$ by $d \varphi$ and $\Delta p$ by $(-d p)$, thus reducing equation (9) to a differential equation-

$$
\frac{-d p}{p-\beta P}=g\left[I-\frac{h}{(p-\beta P)^{\frac{I}{n}}}\right] d \varphi
$$


which may be integrated by substituting $(p-\beta P)^{\frac{x}{n}}=y$.

Starting at $\varphi=0$ when $p=P$, and integrating up to $\varphi, p$, we obtain the equation

$$
\frac{g}{n} \varphi=\log _{e} \frac{(P-\beta P)^{\frac{I}{n}}-h}{(p-\beta P)^{\frac{I}{n}}-h}
$$

Equation (II) is the exact relation between the pressure $p$ and the total piston displacement $\varphi$, when $\varphi$ is composed of an infinite number of infinitesimal steps. It will be nearly correct if $\varphi$ consists of a large number $N$ of small steps of volume $v$. Hence we get an approximate result by substituting $N v$ for $\varphi$; and upon solving for $N$, we have the equation

$$
N=\frac{n}{g v} \log _{e} \frac{(P-\beta P)^{\frac{I}{n}}-h}{(p-\beta P)^{\frac{I}{n}}-h}
$$

which tells us the number of pump cycles or suction strokes $N$ required to reduce the pressure in the $\operatorname{tank}$ from $P$ to $p$.

If we introduce the degree of exhaustion $x=p / P$ and change to common logarithms, equation (I2) may be written in the more convenient form

$$
N=A \log _{10} \frac{C}{(x-\beta)^{\frac{x}{n}}-B}
$$

where

$$
\left.\begin{array}{l}
A=\frac{2.3026 n \theta V}{(\mathrm{I}+\epsilon) \theta_{0} v} \\
B=\frac{\epsilon}{\mathrm{I}+\epsilon}(\mathrm{I}+\alpha)^{\frac{I}{n}} \\
C=(\mathrm{I}-\beta)^{\frac{\mathrm{I}}{n}}-B .
\end{array}\right\}
$$

To find $l$, the lowest degree of exhaustion obtainable, we set $N=\infty$ or $(l-\beta)^{\frac{1}{n}}-B=0$, whence

$$
l=B^{n}+\beta
$$

The values of $v, \epsilon, \alpha$, and $\beta$ are determined by the design of the pump and the adjustment of the valves. The temperature $\theta_{0}$ of the air in the tank depends on the outside conditions. The temperature $\theta$ of the air in the cylinder at the end of a suction stroke depends on the conditions that determine $\theta_{0}$ and also on the running conditions; that is, on the speed and the amount of 
cooling. The value of $n$ depends on the same circumstances as determine $\theta_{0}$ and $\theta$.

When these are all given, together with the volume $V$ which is to be exhausted, $A, B$, and $C$ are known constants, and equation (I3) may be used for computing $N$ for a given $x$. It is rather laborious to use equations (I3) and (I4) for a single computation, but after the values of $A, B$, and $C$ have been computed it is a very simple matter to use equation (13) for obtaining a curve $N=f(x)$, which will obviate the need of further computations so long as the conditions remain unaltered.

It may be noted that $\theta_{0}, \theta, v$, and $V$ occur only in the coefficient $A$, and that $N$ is directly proportional to the value of $\theta V / \theta_{0} v$. Hence if a curve has been constructed for given values of $\epsilon, \alpha, \beta$, $n$ and any value of $\theta V / \theta_{0} v$, it may be used directly for any other value of $\theta V / \theta_{0} v$ by merely multiplying the values of $N$ by a constant factor.

\section{APPROXIMATE FORMS OF THE EQUATION.}

Since the clearance $\epsilon$ and the load on the discharge valve $\alpha$ are always small, the value of $B$ is also small. Hence only a small error can be caused by setting $\alpha=0$, at all events until the denominator in equation (I3) becomes very small; that is, the number of strokes very large. Thus the first part of the curve is very little affected by the value of $\alpha$.

The load $\beta$ on the suction valve should, of course, be made as small as practicable because it slows down the process and raises the limiting degree of exhaustion attainable, as is seen from equation (I5). If the suction valve is operated mechanically and the pump is not run too fast, the value of $\beta$ may be made negligible.

If $\alpha=\beta=0$, equation (I3) reduces to the form

where

$$
N_{1}=A \log _{10} \frac{C_{1}}{x^{\frac{x}{n}}-B_{1}}
$$

$$
\left.\begin{array}{l}
B_{1}=\frac{\epsilon}{\mathrm{I}+\epsilon} \\
C_{1}=\mathrm{I}-B_{1} .
\end{array}\right\}
$$

In this case, the limiting degree of exhaustion is given by the equation

$$
l=B_{1}{ }^{n}=\left(\frac{\epsilon}{I+\epsilon}\right)^{n}
$$

and depends only on the clearance ratio $\epsilon$. 
The ideally perfect pump has no clearance as well as no load on either valve, and is represented by the oil-sealed vacuum pump with positively operated suction valve. For such a pump $\epsilon=\alpha=\beta=0$ and equation (13) reduces to the very simple form

where

$$
N_{0}=A_{0} \log _{10} \frac{\mathrm{I}}{x}
$$

$$
A_{0}=2.3026 \frac{\theta V}{\theta_{0} v}
$$

The limit of exhaustion of such a pump is $l=0$; but when a sealing liquid is used to eliminate the clearance, the vapor pressure of the liquid prevents absolutely perfect exhaustion.

\section{NUMERICAL EXAMPLES.}

To illustrate the use of the foregoing equations we may assume the following data:

Ratio of tank volume to piston displacement................. V/v=1000

Temperature in $\operatorname{tank} 80^{\circ} \mathrm{F}$; in pump $105^{\circ} \mathrm{F} \ldots \ldots \ldots \ldots \ldots \ldots \ldots \ldots .6 / \theta_{0}=1.046$

Exponent of the compression line .......................... $n=\mathrm{r} \cdot 3$

Barometric pressure......................... $P=30$ inches mercury

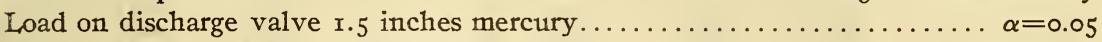

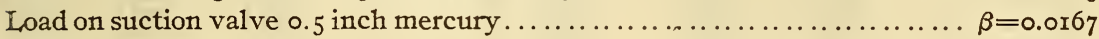

Clearance, 3 per cent..................................... $\epsilon=0.03$

Using these data in equation (I4) we have

$$
A=3040, \quad B=0.03024, \quad \log _{10} C=\overline{\mathrm{I}} .98087 .
$$

So that the particular form of equation (1 3 ) for this case is

$$
N=3040\left\{\bar{I} .98087-\log _{10}\left[(x-0.0167)^{\frac{x}{x \cdot 3}}-0.03024\right]\right\}
$$

and the limit of exhaustion is $l=0.02725=\mathrm{r} / 36.7$. Values of $N$ computed from equation (2I) are given in Table $\mathrm{I}$.

If both valves are operated positively or are so lightly loaded that $\alpha$ and $\beta$ are negligible, while all the other data remain unchanged, we have by equation ( 17 )

$$
A=3040, \quad B_{1}=0.02913, \quad \log _{10} C_{1}=\overline{\mathrm{r}} .987 \mathrm{r} 6
$$

Equation (I6) assumes the particular form

$$
N_{1}=3040\left[\overline{\mathrm{I}} .98716-\log _{10}\left(x^{\frac{x}{1.3}}-0.02913\right)\right]
$$


and the limit of exhaustion is $l=0.01008=1 / 99$. Values of $N_{1}$ are given in Table $\mathrm{I}$.

If the clearance also is zero, so that we have the ideal pump, we have by equations (19) and (20)

$$
N_{0}=2408 \log _{10} \frac{I}{x}
$$

the limit of exhaustion being $l=0$. Values of $N_{0}$ are given in Table I.

It is evident from the table that valve loads such as here assumed do not have much effect on the rate of exhaustion until the process has gone rather far, although they increase the ultimate pressure attainable in the ratio 2.7 to $\mathrm{I}$, in this case. The effect of the 3 per cent clearance, shown by the difference of $N_{1}$ and $N_{0}$, is much less than that of the valve loads shown by the difference between $N$ and $N_{1}$.

TABLE 1.

\begin{tabular}{|c|c|c|c|c|c|c|c|}
\hline$\frac{p}{p}=x$ & $\stackrel{N}{N}$ & $\begin{array}{c}N_{1} \\
\text { equation (22) }\end{array}$ & equation (23) & $\frac{p}{P}=x$ & $\underset{\text { equation (21) }}{N}$ & $\frac{N_{1}}{\text { equation(22) }}$ & $\frac{N_{0}}{\text { equation (23) }}$ \\
\hline $\begin{array}{r}0.9 \ldots \ldots \ldots \ldots \\
.8 \ldots \ldots \ldots \ldots \\
.7 \ldots \ldots \ldots \ldots \\
.6 \ldots \ldots \ldots \\
.5 \ldots \ldots \ldots\end{array}$ & $\begin{array}{l}112 \\
239 \\
383 \\
551 \\
752\end{array}$ & $\begin{array}{l}110 \\
234 \\
375 \\
538 \\
732\end{array}$ & $\begin{array}{l}110 \\
234 \\
373 \\
534 \\
725\end{array}$ & $\begin{array}{l}0.07 \ldots \ldots \ldots \\
.05 \ldots \ldots \ldots \\
.04 \ldots \ldots \ldots \\
.03 \ldots \ldots \ldots \\
.028 \ldots \ldots\end{array}$ & $\begin{array}{l}3369 \\
4103 \\
4799 \\
6741 \\
8475\end{array}$ & $\begin{array}{l}2999 \\
3490 \\
3792 \\
4270 \\
4396\end{array}$ & $\begin{array}{l}2781 \\
3132 \\
3366 \\
3667\end{array}$ \\
\hline $\begin{array}{l}.4 \ldots \ldots \ldots \ldots \\
.3 \ldots \ldots \ldots \ldots \\
.2 \ldots \ldots \ldots \ldots \\
.15 \ldots \ldots \ldots \ldots \\
.10 \ldots \ldots \ldots \ldots\end{array}$ & $\begin{array}{l}1002 \\
1333 \\
1821 \\
2191 \\
2767\end{array}$ & $\begin{array}{r}972 \\
1284 \\
1736 \\
2064 \\
2547\end{array}$ & $\begin{array}{r}958 \\
1259 \\
1683 \\
1984 \\
2408\end{array}$ & $\begin{array}{l}.0275 \ldots \ldots \\
.02725 \ldots \ldots \\
.02000 \ldots \\
.01008 \ldots \\
.00000 \ldots\end{array}$ & $\begin{array}{c}10031 \\
\infty \\
\ldots \ldots \ldots\end{array}$ & $\begin{array}{r}4430 \\
4447 \\
5763 \\
\infty\end{array}$ & $\begin{array}{l}3767 \\
4092 \\
4807 \\
\infty^{487}\end{array}$ \\
\hline
\end{tabular}

WASHINGTON, August 29, 1922. 